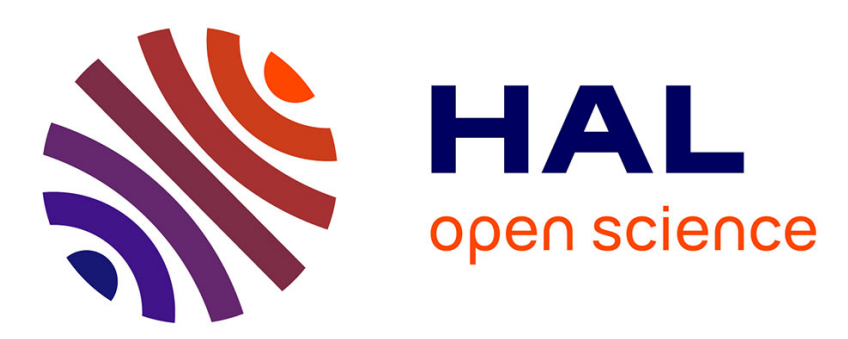

\title{
Automated quantification of the epidermal aging process using in-vivo confocal microscopy
}

Julie Robic, A Nkengne, Benjamin Perret, Michel Couprie, Hugues Talbot

\section{To cite this version:}

Julie Robic, A Nkengne, Benjamin Perret, Michel Couprie, Hugues Talbot. Automated quantification of the epidermal aging process using in-vivo confocal microscopy. International Symposium on Biomedical Imaging, Apr 2016, Prague, Czech Republic. 10.1109/ISBI.2016.7493486 . hal-01363469

\section{HAL Id: hal-01363469 \\ https://hal.science/hal-01363469}

Submitted on 9 Sep 2016

HAL is a multi-disciplinary open access archive for the deposit and dissemination of scientific research documents, whether they are published or not. The documents may come from teaching and research institutions in France or abroad, or from public or private research centers.
L'archive ouverte pluridisciplinaire HAL, est destinée au dépôt et à la diffusion de documents scientifiques de niveau recherche, publiés ou non, émanant des établissements d'enseignement et de recherche français ou étrangers, des laboratoires publics ou privés. 


\title{
AUTOMATED QUANTIFICATION OF THE EPIDERMAL AGING PROCESS USING IN-VIVO CONFOCAL MICROSCOPY
}

\author{
J. Robic ${ }^{\star}$, A. Nkengne $e^{\star}$, B. Perret ${ }^{\dagger}$, M. Couprie ${ }^{\dagger}$, and H. Talbot ${ }^{\dagger}$ \\ * Clarins Laboratories, Pontoise, France. \\ † Université Paris-Est, LIGM UMR 8049, ESIEE Paris, France.
}

\begin{abstract}
Reflectance confocal microscopy (RCM) is a powerful tool to visualize the skin layers at cellular resolution. The epidermal layer appears as a honeycomb pattern, whose regularity decreases with age. Our aim is to provide a method to automatically quantify the regularity of the honeycomb pattern. The proposed strategy relies on a cell-level supervised classification as regular or irregular using spatial information given by a prior segmentation. The aggregated scores defined by the classification results show significant correlation with chronological aging and photo-aging. Thus, our method enables practitioners to more objectively assess the quality of the epidermal layers on large cohort of subjects.
\end{abstract}

Index Terms - Reflectance confocal microscopy, skin aging, image segmentation, classification

\section{INTRODUCTION}

The characterization of skin conditions is a major challenge for skin aging understanding and cosmetic research. Skin aging is defined by a set of alterations of its various components over the years. It is governed by intrinsic and extrinsic parameters. The two major layers of the skin, the epidermis and the dermis, are both significantly affected by skin aging.

The epidermis is the outer layer of the skin, which serves as a physical and chemical barrier against the environment. Cells start from the lower layer of the epidermis and migrate upward to the outer layers. The cells mature throughout this process. They become larger and ultimately end up as a compact anucleated natural moisture barrier. The cell desquamation on the skin surface is compensated by the renewal of the epidermis, a process undertaken by the keratinocytes. With skin aging, the keratinocytes show increased variability in size and shape.

Reflectance confocal microscopy is a powerful tool for non-invasively assessing the skin architecture and cytology. RCM image resolution is similar to routine histology. The epidermal keratinocytes appear as outlined

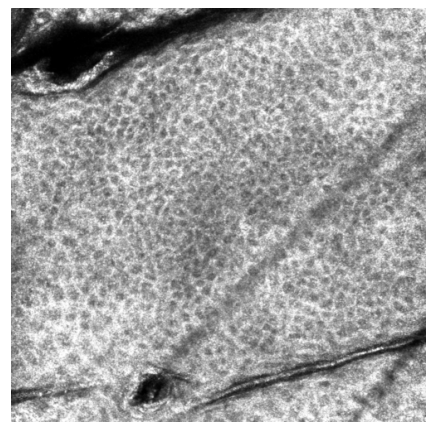

(a) Young epidermis

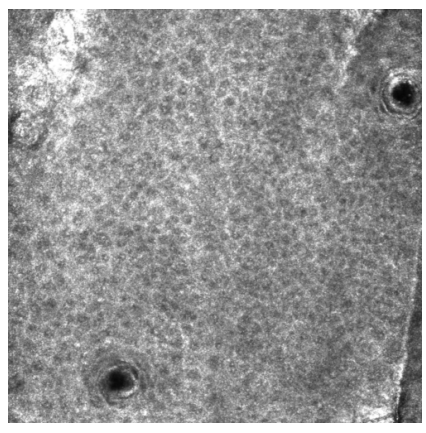

(b) Aged epidermis
Fig. 1. Epidermal honeycomb pattern. One can notice that the images are affected by several defects: noise, blur, non-homogeneous intensity, and artifacts.

cells, which form a honeycomb-like pattern.

Confocal descriptors on skin aging have been identified on RCM images and correlated with histological data [1]. A semi-quantitative aging score was established, requiring visual assessment of the images by experienced dermatologists [2]. One of the descriptors defined by the dermatologists measures the distortion of the honeycomb pattern which progressively loses its regularity with aging, see Fig. 1.

Characterization of the honeycomb pattern has been studied on corneal cells using specular microscopy in $[3,4]$ and RCM in [5]. However, the honeycomb pattern of the corneal cells is much more contrasted and regular than the epidermal.

In this paper, we provide a method for automatically quantifying the regularity of the epidermal honeycomb pattern using in vivo reflectance confocal microscopy. We first define our image acquisition protocol and reference dataset (Sec 2). Then, we propose an algorithm composed of three steps (Sec 3): 1) the image is segmented in individual cells, 2) each cell is classified as regular or irregular by a classifier based on spatial features computed on the region adjacency graph of the cells, and 3) aggregated measures are defined to provide a global score for each image. Our main result is a signif- 


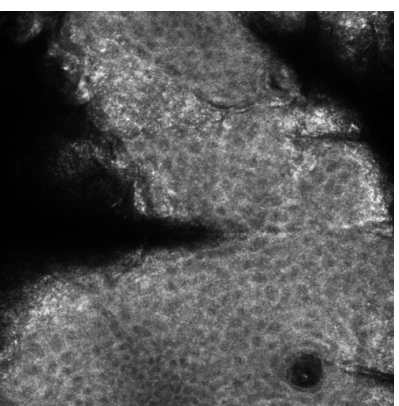

(a) Initial Image

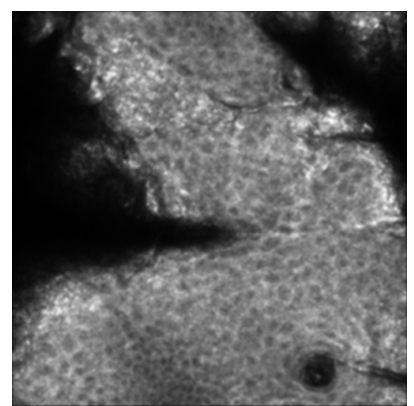

(b) Bilateral filtering

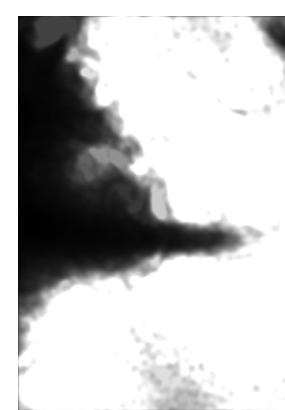

(c) Reconstruction by dilation
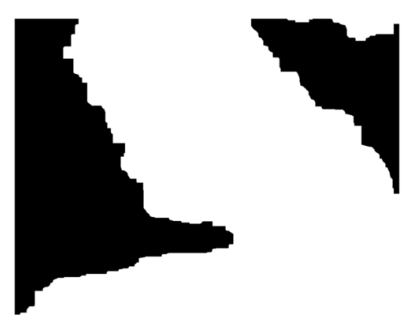

(d) Otsu threshold

Fig. 2. Artifacts detection using connected reconstruction.

icant positive correlation between the proposed scoring and epidermal aging (Sec. 4).

\section{SUBJECTS \& INSTRUMENTS}

Fifteen healthy volunteers with fair skin were enrolled in this study. Volunteers were assigned to two groups: a 7-person group aged from 18 to 25 and another 8-person group aged from 55 to 65 . Our investigations were carried out on the cheek to assess chronological aging, the dorsal forearm and the volar arm to assess photo aging. No cosmetic products or skin treatment were allowed on the day of the acquisitions. Appropriate consent was obtained from all subjects before imaging.

RCM images are acquired using a near-infra-red reflectance confocal laser scanning microscope (Vivascope 1500; Lucid Inc, Rochester, NY, USA) [6]. RCM provides a representation of the skin at the cellular level in a layer set with a thickness less than $5.0 \mu \mathrm{m}$, with melanin and keratin working as natural contrast agents [7]. Each image corresponds to a horizontal section with a $500 \times 500 \mu \mathrm{m}$ field of view and a resolution of $1000 \times 1000$ pixels. On each imaged site, three stacks are acquired from the skin surface to the reticular dermis with a step of $5 \mu \mathrm{m}$. We study the epidermal organization in the spinous layer, i.e. images from $20 \mu \mathrm{m}$ to $40 \mu \mathrm{m}$. In each stack, four images are analyzed.

\section{METHOD}

\subsection{Artifacts Removal}

The skin surface is composed of several structures such as hair, pores and skin topography which influence the image formation at deeper layers, distorting and shadowing the honeycomb pattern below them. Artifacts locations are virtually unchanged though all depths layers. Thus, we need to extract these artifacts once and use a mask on other images. The detection is performed just under the stratum corneum, at $20 \mu \mathrm{m}$ below the surface, where the artifacts are the most contrasted. We apply a bilateral filter with a large kernel to smooth the image while preserving the edges. In order to merge the brightest areas, we perform a morphological reconstruction by dilation. Then, the reconstructed image is thresholded using a local Otsu threshold. The artifacts detection is presented on Fig. 2.

\subsection{Segmentation of the epidermal cells}

Segmentation of the epidermal cells from RCM images is an essential step for studying their morphology, distribution and organization regarding the epidermal state. Epidermal cells are polygonal in shape, with dark central nuclei surrounded by refractive white thin cytoplasm.

The watershed transformation was introduced as a tool for segmenting gray scale images and is now used as a fundamental step in many powerful segmentation procedures where a gray-scale image can be viewed as a topological relief. Following the definition given in [8], each pixel is assigned to the closest local minimum by following a steepest path descent. In this formalism, the borders of the regions are defined on the edges of the pixels, thus each pixel is assigned to a unique region. In our case, an edge linking pixels $i$ and $j$ is weighted with the mean of the intensity values of $i$ and $j$.

Due to the large amount of noise and blur, the direct application of the watershed transform leads to oversegmentation. Using prior knowledge of the cell size, which was correlated with histological data, we can set a lower limit on the size of a cell at roughly $250 \mu \mathrm{m}^{2}$ or 500 pixels in the dataset. We used an area closing to suppress the non-relevant minima measuring less than 500 pixels. An example of segmentation is given in Fig. 3.

\subsection{Classification of the epidermal cells}

After the segmentation of the keratinocytes, each cell is classified as regular or irregular based on its shape 

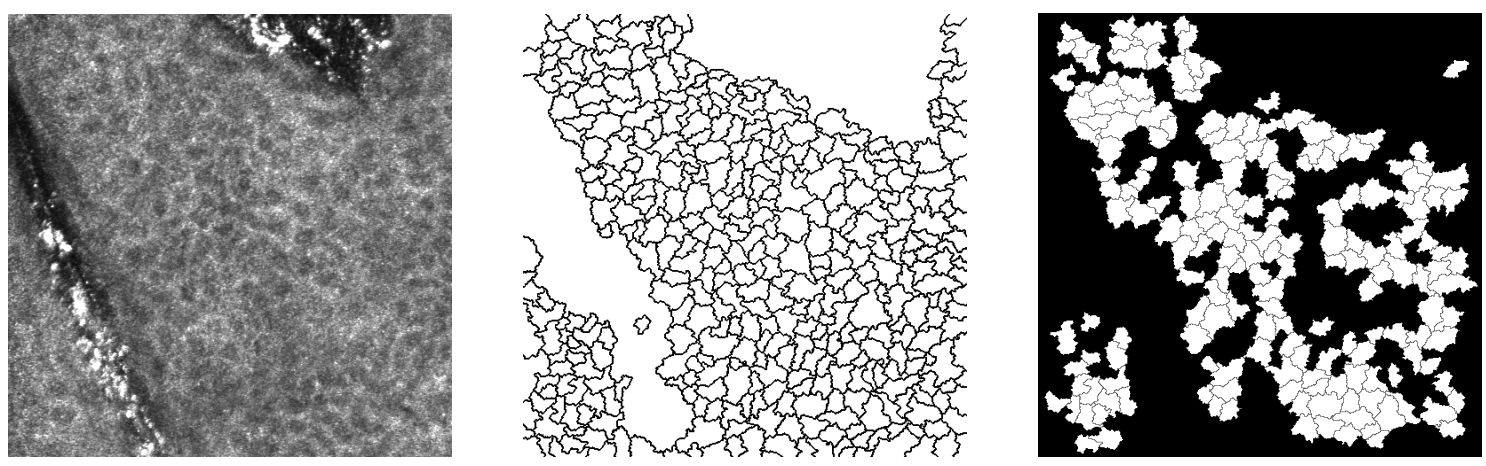

Fig. 3. Epidermal cell segmentation, from left to right: initial image, segmentation, and regular cells class.

and neighborhood. We use classical features from the literature $[3,5]$ :

1. area: the number of pixels in the cell;

2. compacity: $4 \pi \times\left(\right.$ area/perimeter $\left.{ }^{2}\right)$;

3. elongation: $\lambda_{1} / \lambda_{2}$ with $\lambda_{1}$ and $\lambda_{2}$ the eigenvalues of the inertia matrix of the region;

4. number of neighbors (computed from the region adjacency graph);

5. average distance between cells centroids.

In order to perform a supervised classification, a training set was constructed by an expert who labeled about 1500 cells taken at four different depths. The training set was constructed so that it contained roughly the same number of regular and irregular cells.

Since the size of the cells is depth-dependent, we train one Random Forest classifier for each depth. The four classifiers achieved a score better than $90 \%$ on a 10 -fold cross-validation test. An example of the classification of regular cells is presented in Fig. 3. The most discriminant features along with their inter-trees variability are presented in Fig. 4.

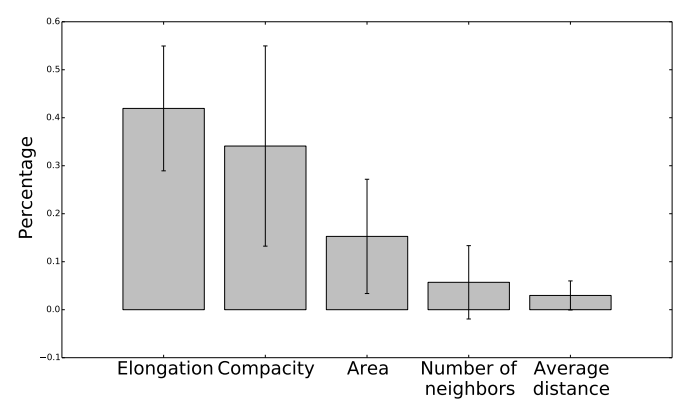

Fig. 4. Feature importance in the Random Forest classifier.

\subsection{Regularity measures}

So far, image scoring has been performed only by experienced dermatologists, who estimate the proportion of irregular honeycomb pattern [2]. We propose to assess the irregularity of the honeycomb pattern using these indicators:

$$
\begin{aligned}
& \text { 1. WDC }=\frac{\text { Number of well defined cells }}{\text { Total number of cells }} \\
& \text { 2. WDR }=\frac{\text { Number of well defined cells }}{\text { Number of connected components }}
\end{aligned}
$$

\section{RESULTS}

Results from the method are expressed as mean \pm one standard deviation. A non-parametric Wilcoxon test is used to compare the two groups of volunteers and image locations. $P$-values of less than 0.05 were considered significant. In addition, Fig. 5 shows box-and-whisker plots of all the measurements at all depths. Similar results were found when comparing depths pairs. WDC were significantly higher in the younger group $(0.59 \pm 0.05)$ than in the older group $(0.57 \pm 0.04)$ on the cheek and volar arm $(0.61 \pm 0.03$ for the younger group and $0.59 \pm$ 0.03 for the older group) but did not show any changes in other locations. WDR were significantly higher in the younger group on the cheek and volar arm. In the younger group, WDR were significantly higher on the volar arm $(10.8 \pm 2.3)$ than on the dorsal arm $(8.4 \pm$ 2.6). No significant difference were seen on the dorsal forearm, which tends to provide credence to the fact that the distribution difference on the other areas are indeed significant.

Finally, in order to test if our method is able to predict the class of a subject, we trained a Random Forest classifier and a SVM classifier on the 15 subjects. Each subject was characterized by its mean score value on the cheek and volar arm. A leave-one-out cross-validation gives an average precision score of $73 \%$ of good classification with the Random Forest classifier and a score of 
$67 \%$ with the SVM classifier.
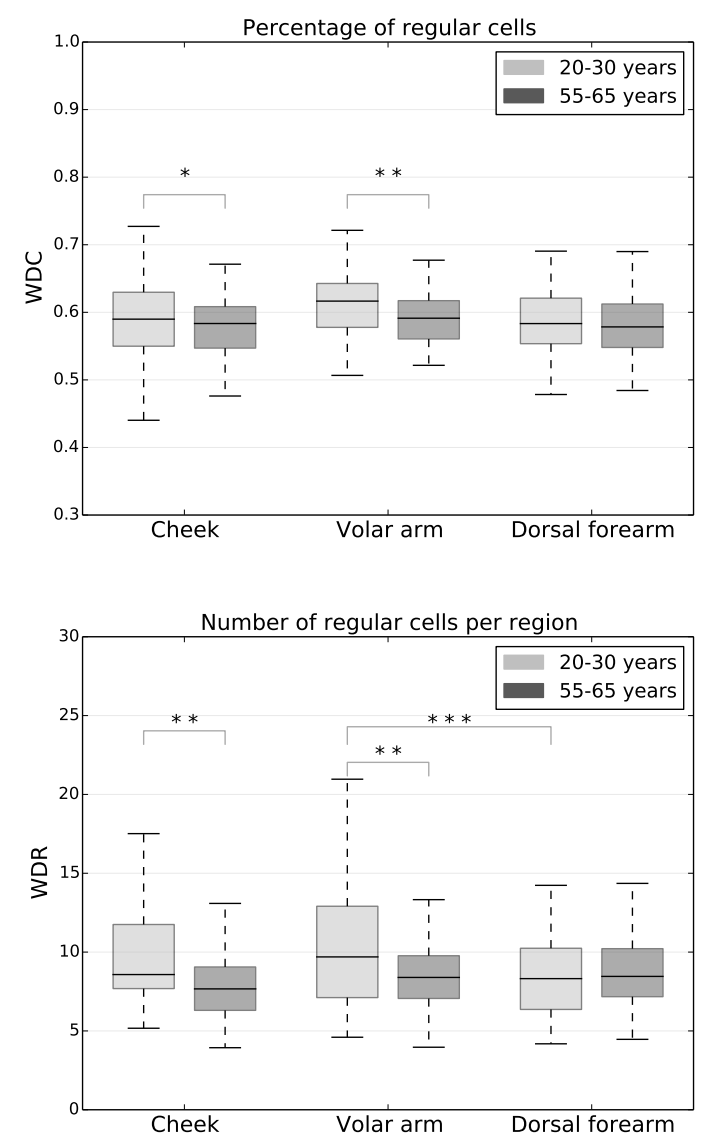

Fig. 5. Results : * $: 0.01<P$-values $\leq 0.05 ;^{* *}: 0.001$ $<P$-values $\leq 0.01 ; * * *: P$-values $\leq 0.001$.

\section{DISCUSSION}

Our results show significant differences between young and old populations, most significantly on the Volar arm. The percentage of regular cells remains stable with age and location, unlike the average size of well-defined regions. This may indicate that there is a link between region-forming in the keratinization process and mechanical properties of the epidermis. The honeycomb pattern irregularity was increased by $22 \%$ in sun-exposed area among young subjects, which indicates that irregular honeycomb pattern due to sun exposition can potentially be quantified using our method. To support this, in previous studies [9], it was found that irregular honeycomb pattern correlates with chronological aging and was exacerbated on UV-exposed areas. The classification results of our subjects in age groups are encouraging, given the small size of our population and intra-class variability. The inclusion of new measures of the epidermal state for aging characterization should allow us to further improve those results in future work. Our next aim will be to develop a skin health measure that correlates well with dermatologist scores.

\section{REFERENCES}

[1] C. Longo, A. Casari, F. Beretti, A.M. Cesinaro, and G. Pellacani, "Skin aging: In vivo microscopic assessment of epidermal and dermal changes by means of confocal microscopy," JAAD, vol. 68, no. 3, pp. e73-e82, 2013.

[2] C. Longo, A. Casariand, B. Pace, S. Simonazzi, G. Mazzaglia, and G. Pellacani, "Proposal for an in vivo histopathologic scoring system for skin aging by means of confocal microscopy," Skin Research and Technology, vol. 19, no. 1, pp. e167-e173, 2013.

[3] L. Vincent and B.R. Masters, "Morphological image processing and network analysis of cornea endothelial cell images," in San Diego'92. SPIE, 1992, pp. 212226 .

[4] Y. Gavet and J.C. Pinoli, "Visual perception based automatic recognition of cell mosaics in human corneal endothelium microscopy images," ISS, vol. 27, no. 1, pp. 53-61, 2011.

[5] D.V. Patel and C.N. McGhee, "Quantitative analysis of in vivo confocal microscopy images: a review," Surv ophthalmol, vol. 58, no. 5, pp. 466-475, 2013.

[6] P. Calzavara-Pinton, C. Longo, M. Venturiniand, R. Sala, and G. Pellacani, "Reflectance confocal microscopy for in vivo skin imaging," Photochem Photobiol, vol. 84, no. 6, pp. 1421-1430, 2008.

[7] M. Rajadhyaksha, M. Grossman, D. Esterowitz, R.H. Webb, and R.R. Anderson, "In vivo confocal scanning laser microscopy of human skin: melanin provides strong contrast," JID, vol. 104, no. 6, pp. 946952, 1995.

[8] J. Cousty, G. Bertrand, L. Najman, and M. Couprie, "Watershed cuts: Minimum spanning forests and the drop of water principle," IEEE PAMI, vol. 31, no. 8, pp. 1362-1374, 2009.

[9] E.M.T. Wurm, C. Longo, C. Curchin, H.P. Soyer, T.W. Prow, and G. Pellacani, "In vivo assessment of chronological ageing and photoageing in forearm skin using reflectance confocal microscopy," BJD, vol. 167, no. 2, pp. 270-279, 2012. 